\title{
PECULIAR ABUNDANCES IN CV SECONDARY STARS: REVISING THEIR EVOLUTIONARY HISTORY?
}

\author{
Thomas E. Harrison, ${ }^{1}$ Steve B. Howell, ${ }^{2}$ Joni J. Johnson, ${ }^{1}$ Heather L. Osborne, ${ }^{1}$ and Derek Homeier ${ }^{3}$
}

We have recently completed an infrared (Kband) spectroscopic survey of two dozen cataclysmic variables. The systems range in orbital period from $P_{\text {orb }}=1.35 \mathrm{hr}$ (EF Eri), to $P_{\text {orb }}=11.0 \mathrm{hr}(\mathrm{V} 442 \mathrm{Cen})$. We detect the secondary star in every system.

Nearly all of the CVs in which we can clearly see the secondary have weak CO features. Most show evidence for enhanced levels of ${ }^{13} \mathrm{CO}$. In addition, we see apparent enhancements and/or deficits of such species as sodium, calcium, titanium, magnesium, silicon, and iron. In addition, we find a few lines that we have been unable to identify (such as the strong feature we call "cataclysmicum" at $2.2767 \mu \mathrm{m}$ seen in the spectrum of EF Eri, and two other very short period CVs). These peculiar abundance patterns suggest that the secondary stars of CVs have nuclear-processed material in their atmospheres.

One prominent example is $\mathrm{U}$ Gem. In Fig. 1 we present the K-band spectrum of U Gem obtained using SPEX on the IRTF. The spectrum of U Gem is perfectly consistent with that of an M4V, except for the extreme weakness of its $\mathrm{CO}$ features (the series of absorption features beginning at $2.29 \mu \mathrm{m}$ in the M-dwarf spectra). In addition, there is a suggestion of ${ }^{13} \mathrm{CO}$ first overtone absorption at $2.345 \mu \mathrm{m}$ in the spectrum of $U$ Gem. Note that the ${ }^{13} \mathrm{CO}$ feature in $\mathrm{U}$ Gem is roughly appropriate for an $\mathrm{M} 4 \mathrm{~V}$ classification-but not for one where the ${ }^{12} \mathrm{CO}$ features are so weak! The decline in the continuua of the late-type stellar spectra at the red end (at $\lambda \geq 2.25 \mu \mathrm{m}$ ) of the $\mathrm{K}$-band is due to water vapor absorption. The decline in U Gem's spectrum suggests that its oxygen abundance is normal. We have used the non-LTE atmosphere PHOENIX to generate a preliminary model for U Gem shown in Fig. 1. In this model, we have set the carbon abundance to $10^{-2} \times$ solar. The weak ${ }^{12} \mathrm{CO}$ features are well reproduced by this model, suggesting an extreme carbon

\footnotetext{
${ }^{1}$ New Mexico State University, Box 30001/MSC4500, Las Cruces, NM 88003 USA.

${ }^{2}$ WIYN Observatory and National Optical Astronomy Observatories, (50 North Cherry Avenue, Tucson, AZ 85726 USA.

${ }^{3}$ Dept. of Physics and Astronomy, University of Georgia, Athens, GA 306027 USA.
}

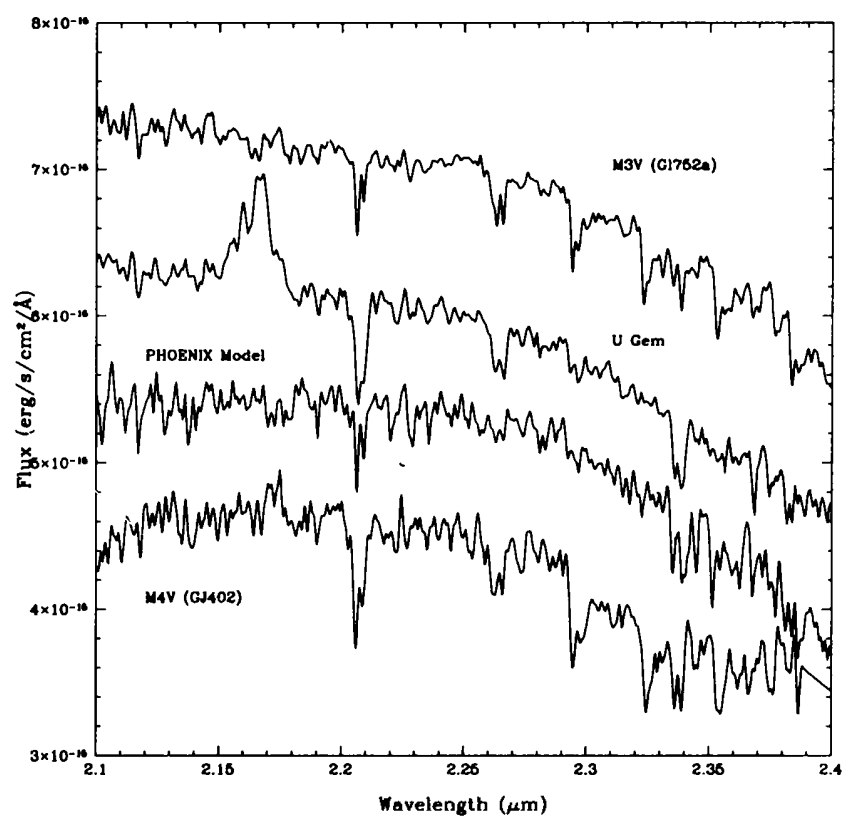

Fig. 1. The infrared spectrum of U Gem (green) compared to those of an M3V (blue) and M4V (red). The black spectrum is a model constructed using the PHOENIX code that has its carbon abundance set to $10^{-2}$ solar.

deficit in the secondary star of U Gem.

From UV spectra, Sion et al. (1998) and Long \& Gilliland (1999) found that the nitrogen abundance for the white dwarf in U Gem was $4 \times$ solar, while the carbon abundance was $0.1 \times$ solar. Models by Marks \& Sarna (1998) could reproduce this abundance pattern by assuming that the secondary star starts off with a higher mass than we observe now. This star could then have begun to evolve off of the main sequence before the secondary contacted its Roche lobe. The mass transfer phase then strips the outer atmosphere of the secondary star, revealing layers where the CNO cycle was operating. During the CNO cycle, the nitrogen abundance increases, while the carbon abundance decreases. The spectrum of $U$ Gem indicates that the standard picture for the evolutionary history for CVs needs revision.

\section{REFERENCES}

Long, K. \& Gilliland, R. L. 1999, ApJ, 511, 916 Marks, P B. \& Sarna, M. J. 1998, MNRAS, 301, 699 Sion, E. M., et al. 1998, ApJ, 496, 449 